\title{
Strategies for the use of immune checkpoint inhibitors
}

\author{
Noriomi Matsumura ${ }^{1}$
}

Published online: 3 September 2021

(c) The Japan Society of Clinical Oncology 2021

This issue contains three reports in which immune check point inhibitors (ICI) were useful in specific conditions.

First, Asahi et al. reports on three patients with liver metastases from non-cutaneous melanoma who were treated with hepatic resection and ICI therapy and were able to survive for a long time [1].

Toyata et al. reports a case of a 69-year-old man with stage IV gastric cancer with peritoneal dissemination [2]. The patient was initially treated with two lines of chemotherapy, but the tumor remained. After treatment with nivolumab, the peritoneal dissemination completely disappeared and curative gastrectomy was possible in this patient.

Sejima et al. reports a case of pathologic cure of postoperative local recurrence and liver metastasis of renal cell carcinoma with sarcoma component in a 57-year-old male patient with the combination of nivolumab and ipilimumab [3].

These reports indicate that the ingenious use of ICIs can result in long-term survival in patients with lethal cancer with metastasis.

Recently, ICIs, as single agents or in combination, have been used not only for recurrence but also as adjuvant therapy after initial surgery or as neoadjuvant therapy before surgery [4]. Earlier use of immune checkpoint inhibitors, along with the development of their biomarkers, is expected to contribute to further improvement in patient cure rates.

\section{References}

1. Asahi Y, Kamiyama T, Kakisaka T et al (2021) Hepatectomy and immune checkpoint inhibitor treatment for liver metastasis originating from non-cutaneous melanoma: a report of three cases. Int Canc Conf J. https://doi.org/10.1007/s13691-021-00495-x

2. Toyota Y, Okamoto K, Tanaka N et al (2021) Conversion surgery of Stage IV gastric cancer with peritoneal dissemination after nivolumab. Int Canc Conf J. https://doi.org/10.1007/ s13691-021-00503-0

3. Sejima T, Masago T, Yoshida M et al (2021) Pathological eradication of recurrent metastatic renal cell carcinoma with sarcomatoid component by nivolumab plus ipilimumab combination therapy. Int Canc Conf J. https://doi.org/10.1007/s13691-021-00501-2

4. Le Saux O, Lounici Y, Wajda P et al (2020) Neoadjuvant immune checkpoint inhibitors in cancer, current state of the art. Crit Rev Oncol Hematol. https://doi.org/10.1016/j.critrevonc.2020.103172

Publisher's Note Springer Nature remains neutral with regard to jurisdictional claims in published maps and institutional affiliations.
Noriomi Matsumura

noriomi@med.kindai.ac.jp

1 Faculty of Medicine, Kindai University, Osaka-Sayama, Japan 